\title{
Perceived barriers and facilitators in using text and voice messaging for improving HIV and sexual and reproductive health of female entertainment workers in Cambodia: a qualitative study
}

\author{
Kiyoko Kai Xuan Ong ${ }^{1}$, Janelle Shaina $\mathrm{Ng}^{2}$, Chhorvoin $\mathrm{Om}^{3}$, Pheak Chhoun ${ }^{3}$, Sovannary Tuot ${ }^{3}$, \\ Siyan $\mathrm{Yi}^{1,3,4,5}$
}

${ }^{1}$ Saw Swee Hock School of Public Health, National University of Singapore and National University Health System, Singapore, Singapore; ${ }^{2}$ Wee Kim Wee School of Communication and Information, Nanyang Technological University, Singapore, Singapore; ${ }^{3}$ KHANA Center for Population Health Research, Phnom Penh, Cambodia; ${ }^{4}$ Center for Global Health Research, Touro University California, Vallejo, CA, USA; ${ }^{5}$ School of Public Health, National Institute of Public Health, Phnom Penh, Cambodia

Contributions: (I) Conception and design: KKX Ong, JS Ng, C Om, S Tuot, S Yi; (II) Administrative support: P Chhoun, S Tuot, S Yi; (III) Provision of study materials or patients: P Chhoun, S Tuot; (IV) Collection and assembly of data: K Ong, C Om, P Chhoun; (V) Data analysis and interpretation: KKX Ong, JS Ng, S Yi; (VI) Manuscript writing: KKX Ong, JS Ng, S Yi; (VII) Final approval of manuscript: All authors.

Correspondence to: Siyan Yi. 12 Science Drive 2, \#10-01, Singapore 117549, Singapore. Email: ephsyi@nus.edu.sg.

Background: Female entertainment workers (FEWs) in Cambodia are one of the hard-to-reach populations at risk of human immunodeficiency virus (HIV) and poor sexual and reproductive health (SRH). Due to the stigmatizing nature of their work, it is difficult to reach them with prevention and treatment services. The Mobile Link project is a mobile health (mHealth) intervention that aims to deliver health messages to FEWs and link them up to health services. This study aims to explore the perspectives of stakeholders on the Mobile Link and identify barriers and facilitators to the project implementation, in order to determine areas for improvement of future mHealth interventions.

Methods: This study was conducted between November to December 2018 in the capital city and other three provinces where the Mobile Link was implemented. We employed a qualitative research design using an interpretative approach. A purposive sampling method was used to recruit participants across four project sites. All participants were stakeholders involved directly or indirectly with the Mobile Link. Nine different groups of stakeholders at the national, non-governmental organizations, community, and individual levels were recruited. Seventeen semi-structured in-depth interviews (IDIs) and five focus group discussions (FGDs) were conducted and transcribed. Data were managed using NVivo 12 and analysed using thematic analysis with an inductive approach.

Results: We derived four major themes: (I) perceived benefits, (II) attitudes, (III) access, and (IV) enabling environment. Different levels of stakeholders reported on the perceived benefits of the Mobile Link, including ease of use, knowledge gained, link to services, and cost-effectiveness. Perceived benefits and enabling environment were identified as facilitators to implementation of the project. Barriers included technological issues, operational challenges, poor rapport with entertainment establishment owners, and low motivation to participate in the Mobile Link among FEWs.

Conclusions: The Mobile Link has many advantages and is well-accepted by stakeholders from the national and community levels. We discussed the implications of the perceived facilitators and barriers identified on the project and mHealth interventions. Implications discussed should be taken into consideration by organizations implementing mHealth interventions for HIV key populations in Cambodia as well as in other resource-limited settings.

Keywords: Human immunodeficiency virus (HIV); key populations; mHealth; stakeholders; qualitative study; low- and middle-income countries 
Received: 13 November 2019; Accepted: 30 March 2020; Published: 05 October 2020.

doi: 10.21037/mhealth.2020.04.01

View this article at: http://dx.doi.org/10.21037/mhealth.2020.04.01

\section{Introduction}

Mobile health (mHealth) interventions are health interventions which are delivered primarily to or through participants' mobile devices (1). Literature on mHealth interventions have described them as being convenient, cost-effective, personalized, and more engaging for those who are less inclined to traditional services $(2,3)$. They enable program implementers easier and more private access to especially hard-to-reach or vulnerable populations. Access to health information and linkage to services is easier through mobile phones since they are personal devices that afford conveniences, in terms of portability and privacy because it is typically owned and used by one person $(1,4)$. These two features of mHealth interventions are especially important as HIV key populations are often stigmatized or discriminated against in some way and find it difficult to ask for help upfront (5).

mHealth has been suggested as appropriate in improving access to healthcare in developing countries with limited resources $(6,7)$. In Cambodia, mHealth has already been successfully used in the areas of managing chronic diseases such as diabetes and hypertension, tuberculosis, and post-abortion contraception (8-10). Another important target group for mHealth intervention in Cambodia is female entertainment workers (FEWs). FEWs work in entertainment establishments such as beer gardens, karaoke lounges, massage parlors, and restaurants $(11,12)$. It is estimated that there are approximately 52,500 FEWs in Cambodia, and this number has been increasing $(13,14)$. FEWs may engage in direct and indirect sex work, putting them at high-risk for HIV and other sexually transmitted infections (STIs) (15). The HIV prevalence of FEWs was found to be $3.2 \%$ in 2014 , 5 -fold higher than that of the general adult population in the country $(16,17)$. The incidences of STIs such as chlamydia trachomatis, buman papillomavirus, and neisseria gonorrboeae among FEWs are $11.5 \%, 41.1 \%$, and $7.8 \%$, respectively (15). Unwanted pregnancies and induced abortions are also common among FEWs, with $35 \%$ reported having experienced at least one pregnancy, and $28.5 \%$ reported having experienced at least one induced abortion since working in entertainment establishments (11).
In Cambodia, a wide range of free HIV and sexual and reproductive health (SRH) services are provided by the government and community-based non-government organizations (NGOs) (15). Despite this, a recent study found that only $52.3 \%$ of FEWs had been tested for HIV in the last 6 months, far from the goal of $90 \%$ HIV testing in key populations set by the national HIV program (18). This could be due to the change in setting, as FEWs are now "underground", which makes it harder to reach them with prevention and treatment services (19). The high stigma surrounding FEWs' nature of work causes them to avoid seeking HIV and SRH services for fear of being identified or discriminated against (20). Therefore, it is important to find a way to reach FEWs in a way that allows them to maintain their privacy and confidentiality. This demonstrates a clear need for education and outreach to help prevent HIV and promote SRH among this population.

Previous surveys show that $93 \%$ of FEWs in Cambodia own a phone, and $28 \%$ own a smartphone (21). This means that an mHealth intervention with this group is unlikely to encounter lack of access to mobile phones as a limitation. FEWs have also expressed their interest in receiving health messages via mobile phone $(22,23)$. Their openness to receiving interventions through this medium also means that engagement and compliance are likely to be higher if an mHealth intervention is to be implemented. This makes an mHealth intervention a suitable method to access and educate FEWs.

The Mobile Link project is an mHealth intervention that aims to educate FEWs on a variety of pertinent health topics (e.g., HIV, SRH, gender-based violence, cervical cancer, substance abuse) and encourage linkage of FEWs with prevention and treatment services through contact with the project staff members. These health messages are sent via short message services (SMS) or voice message (VM) depending on what the FEWs had chosen. Where needed, the Mobile Link staff will connect FEWs to outreach workers under partnerships with community-based NGOs, who will accompany them to these services (15).

mHealth interventions are often not evaluated in resource-limited settings such as Cambodia due to extra costs, making it difficult to determine program 
effectiveness (20). These evaluations are crucial for improving program design as a whole and increasing cost savings where possible. Otherwise intervention efforts remain uninformed and inconsistent. Evaluating the effectiveness of an mHealth intervention consists of several important points. First, acceptability of the program to the target group is important because it gives insights into whether the program will be willingly propagated or sought out among this population. Second, the feasibility of the program is important to understand because it aids program implementers in deciding whether the program can be implemented elsewhere, or should be discontinued for any reason. This component requires perspectives from all the stakeholders who are involved in endorsing, promoting, designing, collaborating, executing, and receiving the program.

In this study, the socio-ecological model will be used as the framework for analyses since it presents a layered perspective that reflects how various stakeholders at each level of society could contribute to the program success (24). Through understanding stakeholders' perspectives, we hope to identify the enablers and barriers in implementing the Mobile Link project so as to target areas for improvement. Accordingly, the present study aims to provide a qualitative assessment of the effectiveness of the Mobile Link so as to identify shortcomings, recommend improvements specific to this program, and provide a scientific scaffold for stakeholders looking to design similar mHealth interventions in resource-limited settings.

\section{Methods}

\section{Study design}

This study was conducted as part of the midterm evaluation of the Mobile Link. Details of the Mobile Link intervention have been published elsewhere (13). A qualitative research study design was employed based on an interpretative approach.

\section{Participant selection}

A purposive sampling method was used to recruit the participants across the four sites of the Mobile LinkBanteay Meanchey, Battambang, Phnom Penh, and Siem Reap. All participants were stakeholders involved directly or indirectly with the Mobile Link. Nine stakeholders were recruited to understand feasibility and acceptability perspectives from each level—national, community, organization, and individual. At the national level, stakeholders endorse and provide political support for the Mobile Link to be conducted. Stakeholders at the community level collaborate to tap on the strengths of the different organizations and expand their network of reach to wider population of FEWs. Within the organization level, multiple stakeholders are involved to plan and execute the Mobile Link. Finally, at the individual level, participating FEWs are the primary beneficiaries of this project and have to engage with the intervention contents and staff in order to truly benefit. As illustrated, an mHealth program such as the Mobile Link requires different levels of society to work together to be successful. Hence, perspectives from stakeholders at each level are crucial for informing program design.

\section{Recruitment}

An email or a phone call was made to stakeholders to request their participation in the study.

\section{Study settings}

Interviews for stakeholders in Battambang and Phnom Penh were conducted at the province itself and at a place that was private and convenient for the participants. Interviews conducted with stakeholders from Banteay Meanchey and Siem Reap were done over the phone instead, due to time and financial constraints.

\section{Instrument development}

In-depth interview (IDI) and focus group discussion (FGD) topic guides were crafted for the different groups of stakeholders. Consultative meetings with the research team and the Mobile Link staff led to multiple revisions of the topic guides. The topic guides were created in English and translated into Khmer for stakeholders who did not understand English. The guides were designed to be semistructured so as to gather the perspectives of stakeholders on the acceptability and feasibility of the Mobile Link, yet allow for other related views that were not specifically covered.

\section{Data collection}

Data collection took place between November to December 2018. Study aims were explained to all participants and 
verbal informed consent was taken prior to commencing the interviews. All interviews were audio-recorded and transcribed verbatim. Those in Khmer were translated to English prior to transcription. Each interview was conducted once. Reflective field notes were taken by researchers right after the interviews. All participants were compensated with a small token of appreciation for their time spent doing the interviews. Seventeen IDIs were done and each interview lasted approximately 15 to 42 minutes. Five FGDs were carried out with outreach workers, the Mobile Link field staff, and FEWs. The FGDs lasted between 46 minutes to 1 hour and 44 minutes. As stakeholders were approached on a voluntary basis, there were no drop-outs.

\section{Data management and analyses}

All personal identifiers were removed and alphanumeric codes were given to transcripts to anonymize participants. Data were coded using NVivo 12 software and analyzed using an inductive approach. Thematic analyses were undertaken guided by the domains of acceptability and feasibility (25). A codebook was derived and the themes and sub-themes surfaced. Independent checking of transcripts was done by an external researcher and agreement for the themes and sub-themes were reached.

\section{Results}

We present our findings under four main themes, categorized broadly under domains of acceptability and feasibility: (I) perceived benefits, (II) attitudes, (III) access, and (IV) enabling environment. The domain of acceptability includes the themes of perceived benefits and attitudes. The first theme discusses perceived benefits of the Mobile Link from the perspectives of FEWs and other stakeholders. The theme of attitudes describes the attitudes of stakeholders towards the Mobile Link.

\section{Perceived benefits}

\section{Ease of use}

The Mobile Link provided FEWs with the option of receiving messages via SMS or VM. Most of the NGO partners felt that the VM function of the Mobile Link was beneficial as it aided those who were unable to read.

"(The) voice message is good for those who cannot read, people cannot read use voice messages." [IDI-4, NGO partner].

FEWs in the FGDs who chose VM welcomed this function also because they felt that listening to the health messages was easier than reading it from the phone.

"I chose voice message, because it's easy to listen. Fust press and listen." [FGD-3, FEWs].

FEWs were often busy with work and did not have time for typical in-person outreach activities conducted by other organizations (e.g., face-to-face group counselling). Having messages sent to their mobile phones allowed them to still benefit from SRH information without being physically present at other modes of outreach.

"Sometimes they don't have enough time you know to spend time with us, but they can just read their mobile phone, and they can get the information easily, yea. [...] I think in terms of time; I mean because you know FEWs (are) very busy and their schedule (is) full at night and then in the morning they sleep, and they have very limited time to access service information, so it's very convenient for them." [IDI-10, MBL Program Team].

\section{Knowledge gained}

Another perceived benefit of the Mobile Link was increasing knowledge. This was a recurrent theme across the different levels of stakeholders-provincial, community, NGOs, and beneficiaries. The health messages designed for the Mobile Link covered a wide variety of pertinent topics such as gynecologic health, cancer, HIV, STIs, condom use, contraception, gender-based violence, and pregnancy. These health messages were regarded as relevant and useful for facilitating engagement with beneficial health behaviors. The Mobile Link field staff reported that FEWs performed testing for HIV and STIs after receiving the health messages.

"If we give out the speech to them, or send out the messages to them, the more we do that... because the women don't have a lot of general knowledge like us. So, the more we provide to them, the women would also... her self-protection skills or her knowledge would be better." [IDI-3, Phnom Penh Municipal Health Department].

"But when we send out those messages, they returned to do blood test [...] Yes. They started to know more... They have knowledge about health, they know how to protect themselves etc." [FGD-2, Mobile Link Field Staff].

One of the FEWs also corroborated that she understood more about her own health and how to better protect herself from STIs because she received the health messages.

"I can get more knowledge and understanding more about bealth, know how to protect after listening the message. I can protect myself more than before [...] I can know more about uterus problem, about transmission diseases, how to protect." [IDI-12, FEW at Siem Reap]. 


\section{Link to services}

Other than receiving health messages, another perceived benefit of the Mobile Link is that FEWs are able to link up with SRH services by calling up the Mobile Link field staff for assistance.

"We get to listen to the messages. And we have infection, something like that. We can call them (refers to Mobile Link staff), ask them to take us to the hospital, and if we want..." [FGD-4, FEWs at Phnom Penh].

The Mobile Link field staff then connect them to outreach workers who advise the FEWs on the nearest relevant health facility and accompany them for treatment, screening, or consultation. This helped FEWs in making decisions about professional assistance.

"If they want to check up for gynecological diseases or something, we give them choices. Like we tell them that the services are available at this NGO clinic, and a referral hospital that is nearer, where do they want to go." [FGD-1, Outreach workers].

\section{Resource efficient}

One provincial health department stakeholder raised the current shortage in HIV funding as a challenge for conducting such interventions.

"Overall, this project is really important. Hmm... and it's also really good to the current situation that we (are) facing the funding shortage and things like that." [IDI-2, Battambang provincial health department].

As funding decreases, mobile phone programs like the Mobile Link project could lead the way forward as an efficient method of delivering health messages and services. The usual outreach activities involved visiting sites every three months and was very time and labor intensive. Comparatively, the Mobile Link was seen as a more efficient way of delivering an intervention because the same information could be delivered directly and more frequently to a larger group via mobile phones.

"Hmm... that's why we say (we) can target more people, not just go to outreach every three months. And hmm... now you know like HIV funding is decreasing, so the resources are also cut. So that project is very what we call efficient in a way that we can reduce resource, streamline the interventions to meet their needs." [IDI-10, Mobile Link Team].

\section{Attitudes}

\section{Rapport with entertainment establishment owners}

The Mobile Link field staff visit FEWs once every month to provide them the USD $\$ 1$ phone card as part of their compensation for participating in the study. They would also ask the FEWs if they had experienced any symptoms which could require health services and if they had been receiving all the health messages. However, the field staff sometimes encountered difficult entertainment establishment owners who refused them entry or limited the time they had with the FEWs. This made the visits hurried and unpleasant, which interfered with the provision of the compensation to FEW participants and health checks.

"Those big karaoke bars are not easy. Sometimes, we asked them, we told them, they still wouldn't let us go in. [...] And for another establishment, if we meet the owner, they just scold us. They scold like, what are you AIDS group coming here for? And when I tried to negotiate with them, because I used to go with an NGO, but they're still being difficult with us. So bow should we do it? Do it quick. We bave to see women quickly." [FGD-2, Mobile Link Field Staff].

\section{FEW' motivation}

Busy work schedules and laziness often robbed FEWs of the motivation to seek health services, especially if nothing was overtly amiss. As a result, they often lapsed back into less time-consuming routines or failed to make time for health checks.

"Sometimes I want to go. But because I was busy with my work, so I was thinking back and forth, then I just felt lazy to go. I want to get uterus check-up, but I don't have anything bad happening, or itchy, just want to clean up. But I don't have time." [FGD-3, FEWs at Phnom Penh].

FEWs described how even the simple task of consistently reading their health messages felt very challenging because of the perceived lack of time and energy after engaging with a full day of work.

"I work till very late at night, till morning, and I come back bome drunk, so I just go to sleep till 1pm or 2pm or 4pm. Sometimes I get to read it, I just read it. Sometimes, I don't as well. I don't always read it every time." [IDI-15, FEW at Phnom Penh].

The Mobile Link field staff also cited that boredom and laziness led to a lack of motivation to continue the program; so some FEWs quit the Mobile Link after some time.

"They just quitted and said I'm lazy. They're bored with those calling, they're lazy to pick up etc." [FGD-2, Mobile Link Field Staff].

The following section discusses findings in the domain of feasibility. It includes the themes of access and enabling environment. The theme of access describes FEWs' access to health information and services, and Mobile Link field 
staff's access to FEWs. The theme of enabling environment describes environmental factors across layers of society that supported the successful execution of the Mobile Link.

\section{Access}

\section{Access to information and services}

Some technological challenges were brought up by different stakeholders. One of the issues was that messages failed to get sent to FEWs' mobile phones. This means that FEWs would not have received the full inventory of messages prepared for them and were missing out on some pieces of health information due to technological inadequacies.

"But for example, we deliver the $1^{\text {st }}$ message on Monday, we sent to you 10 messages. And the system shows the success delivery is 8 messages, and the other 2 are failed. [...] Generally, in one day, in average, in one time, the SMS is sent out around 1500 SMSes. And the failed messages are around 120 to 130 messages." [IDI-11, Technology support staff].

Operational challenges affect FEWs' access to health services. The opening hours of health facilities were perceived to be inconvenient to FEWs. FEWs worked till many hours past midnight, and most spent the morning asleep. Their work day begins again in the afternoon around $3 \mathrm{pm}$. Hence, they were unable to visit the health centers in the afternoon because that would cause them to be late for work. This prevented FEWs from obtaining much needed treatment or screening without undesirable or unfeasible disruption to their work. The possible disruption to their work could also come at a cost, as employers could dock their pay if they were late for work.

"From 7AM till 11AM. And they open again at 1:30PM or 2PM. But it takes quite a while to take the women there, and the women start working at 3PM, so they cannot go with us. [...] If they go to work late, the owner would deduct their payment." [FGD-1, Outreach Workers].

\section{Mobile Link field staffs' access to FEWs}

Frequent changing of phone numbers by FEWs was another operational challenge. Since health messages were only sent to FEWs' registered phone numbers, other phone numbers that they used did not receive the message interventions. Therefore, when FEWs changed their phone numbers without informing Mobile Link staff, the health message intervention was disrupted, and Mobile Link staff would be unable to contact them for follow-ups or updates. Eventually, dropping out of the Mobile Link for this reason was also a possibility.
"They change their number, and the previous number was terminated. [...] When we call that number, that number is unreachable." [FGD-2, Mobile Link Field Staff].

"I think the nature of FEWs is different from general people. They change their phones more frequently, and their work also change back and forth, making the possibility of them changing phone number is high, and therefore the chance that they drop from the project is also bigh." [IDI-9, Mobile Link Coordinator].

\section{Enabling environment}

\section{Political support}

Strong political support was key to successful implementation of the project as endorsement from authorities facilitated other collaborations. There appeared to be good relationship between the implementing organizations and national and provincial level stakeholders.

"We have good collaboration with national level, we have support from the National AIDS Authority, National Center for HIVIAIDS, Dermatology and STD, Ministry of Posts and Telecommunications. We keep them in the loop, share information with them, request for their supports. Our relationships with local authority, especially Provincial Health Department in every province, we see that our model of implementation is well supported." [IDI-9, Mobile Link Coordinator].

\section{Community partnerships}

The success of the Mobile Link intervention also hinged on its partnerships with other NGOs. The NGO partners had good and long-standing relationships with entertainment establishment owners. These warm relations allowed NGO partners to facilitate Mobile Link staff's access to these venues and, by extension, to FEWs working there.

"...together with the Mobile Link through (our) communication with the entertainment establishment owners, (we can) ask for the staff of the Mobile Link to access inside the area. Because we believe that we are the old persons who have the connection with the entertainment establishment owners, so it's easier than the new one who goes to communicate for the first time." [IDI-5, NGO partner].

\section{Financial support}

The Mobile Link staff feel that financial support for SRH services is an important resource for FEWs. These can come in various forms such as insurance or referral cards, due to the different modes of payment required by different health facilities in different provinces. Services 
may also be paid for by the NGO partners when FEWs are accompanied by outreach workers to the health facilities.

Mobile Link field staff 1: "If they go to public hospital, it is free of charge. But they must have referral card from an NGO, then the provincial hospital is free."

Mobile Link field staff 2: "For going to the hospital, if (it is) in Phnom Penh, the services for women are free of charge. Because in Phnom Penh, KHANA partnered with NGOs in Phnom Penh. They are the ones that pay for the services." [FGD-2, MBL Field Staff].

One of the requirements of receiving financial aid for health expenses is that FEWs must identify themselves. Unfortunately, a strong fear of stigma associated with sex work still prevails in Cambodia. Hence, despite the financial support offered, some FEWs still preferred to go to private health facilities and pay for themselves as identifying as a FEW was not necessary.

"They show their identity for the health center, they will have the special discount for. But maybe some do not want to show their identity that they are the entertainment workers. [...] because of the great discrimination in Cambodian society. When people hear that, ab she is a woman working in the karaoke bars, in the massage parlors, the people around noted that they look like sex workers. The concept is like that". [IDI-5, NGO partner].

\section{Discussion}

This study describes and organizes stakeholder perspectives on the acceptability and feasibility of the Mobile Link project, an mHealth intervention aiming to improve HIV and SRH outcomes among FEWs, a hard-to-reach population in Cambodia. Our results support the existing literature on mHealth interventions as being easy to use, convenient, and increasing accessibility to information and services (1-3). Stakeholders felt that the Mobile Link was easy to use and convenient as messages sent were less demanding of FEWs' time as compared to usual outreach activities such as face-to-face counselling and education sessions. FEWs could benefit from increased knowledge with less disruption to their work. Mobile phone-based interventions should be seriously considered as the primary mode for intervention especially when the program lasts for an extended period of time and requires periodic contact with the target population.

VM improved the inclusivity of the Mobile Link by accommodating those with low literacy who could not read texts (22). This improved the usability of the Mobile Link as FEWs reported that it was easy to use and understand.
Future interventions should strongly consider the option to use VM delivery of knowledge to benefit as many recipients as possible regardless of literacy levels.

In order to ensure that the health messages sent would be relevant and comprehensible by FEWs, a participatory approach was employed which involved FEWs through IDIs and FGDs to develop the message bank that would be used for the Mobile Link intervention. Messages were tailored to topics that FEWs were interested in and at a level where they could understand them easily (15). The success of this set of messages was reflected in our findings which illustrated high acceptability of the Mobile Link among FEWs. Where educative messages are central to the objectives of an intervention, a participatory approach to message development should be considered as it seems to produce messages that are comprehensible and engaging for the target audience.

Better knowledge of HIV has been found to be associated with higher HIV testing rate (15). The Mobile Link therefore aimed to provide knowledge through health messages to FEWs. Our findings show that the Mobile Link stakeholders concurred that increasing knowledge through the intervention has been beneficial. FEWs also reported that understanding more about their health enabled them to more effectively protect themselves from HIV and STIs and maintain their SRH. Hence, increasing knowledge should remain a primary goal for interventions that are targeting hard-to-reach populations who may be disadvantaged in terms of education or access to educative resources.

Other factors that may influence HIV testing include socio-demographic factors, perceived threat, services available and its barriers, and social network (26). With an understanding of stigma and discrimination surrounding FEWs, seeking help could be difficult to them. Hence, the Mobile Link sought to provide a linkage between them and health services. Through the linkage to outreach workers, FEWs were provided with the opportunity to seek advice and be connected with relevant services. This in-person guidance was well-received by some FEWs who were able to go for these appointments. Future interventions that have components which require the target group to make decisions about health can consider using this two-pronged (mobile and in-person) approach to provide guidance for appropriate decision moments.

mHealth interventions have been shown to have a wider reach due to high mobile phone penetration in Cambodia. This allows for information to be shared with large groups of people more quickly. The Mobile Link was also perceived 
by stakeholders to be resource efficient, in that the methods used are less labor and time intensive. However, having lesser inputs does not necessarily translate to the same or better outcomes. Our findings remain preliminary on the subject of financial efficiency as this would need to be triangulated with quantitative evidence on improved health knowledge or increased usage of SRH services, which the Mobile Link would be analyzing at a later stage.

Mounting an intervention like the Mobile Link requires a social environment that facilitates close collaboration among stakeholders at various levels of the society (27). Indeed, strong support of our national and community level stakeholders played a crucial role in the Mobile Link's execution. It enabled pooling of social and technological resources that smoothened interpersonal relationships and allowed extended access to FEWs. Stakeholders should be mindful of the cross-cutting collaborations needed to bring necessary resources to bear, as well as the persons needed to facilitate cultivating these relationships.

FEWs' access to health information can be hampered by technological limitations and operational challenges, e.g., messages that are not delivered, as mentioned by our stakeholders. There could be many reasons why messages failed to send such as cell phone network coverage and reliability, or cell phone number changes due to participants migrating for work or other various reasons (21). This has been recognized as a challenge for mobile phone-based interventions in Cambodia and other countries (28-31). Where possible, technology should be further tested for stability before rolling out the interventions.

In Cambodia, FEWs often change phone numbers. As the Mobile Link hinges on delivery of health messages to a designated phone number, this delivery can become interrupted when FEWs switch phone numbers. Future studies should look into ways to improve retention of single phone number use and also how this issue may be mitigated through reminders to FEWs to notify the Mobile Link staff on the change of phone numbers and promptly updating the server. Future interventions should ensure that timely reminders of such changes are sent, and that an alternative mode of contact (e.g., hotline) should be provided in the event that participants change contact numbers.

The issue of accessing health services as cited by our stakeholders was that health facilities opening hours were inconvenient for FEWs. However, it might be difficult to extend hours of health facilities due to the shortage of healthcare workers in Cambodia-1.4 health worker per
1,000 (32). Additionally, because FEWs work in different entertainment establishments with different working hours, it would be difficult to find a common convenient time to access health facilities $(11,17)$. In addition, difficult entertainment establishment owners may not grant FEWs time to access the services and may even dock their pay if they were late for work. While it may not be feasible to extend health facilities opening hours, convincing entertainment establishment owners to protect a day of leave for the FEWs to seek health services is something that can be further explored.

From our findings, motivation of FEWs surfaced as a reason for poor uptake of health services as they often felt lazy or perceived a lack of time to seek services. There could be a multitude of factors that affect health-seeking behaviors such as issues with entertainment establishment owners, inter-personal relationship of FEWs and their partners, or factors like stigma and discrimination which have been reported in other studies (33-35). It would be helpful to further explore what are the key reasons why FEWs do not seek health services and design interventions that can facilitate health-seeking behaviors.

A point to note is that in its present form, the Mobile Link was not designed to address these issues of motivation or rapport with entertainment establishment owners. However, it is clear from our findings that if any mHealth intervention wants to succeed in the future, it would be critical for the implementing NGOs to address these challenges. We also need to recognize that some of these barriers cannot be addressed by NGOs alone, like stigma and discrimination in the general population, which would require a whole-of-society approach.

\section{Limitations of the study}

As not all interviews were conducted in English, and not all interviewees were comfortably fluent in English, there could have been a loss of nuance during interviews. Translation allowed for non-English speaking participants to be interviewed and that reduced bias in our sample. However, translations often fail to capture the original meanings of the speakers in its entirety, which could lead to additional loss of nuance and possible misinterpretation. In future evaluations, it would be ideal to get bilingual Khmer and English speakers to conduct the interviews.

Data saturation was likely not reached as there was small sample size per level of stakeholders, leading to limited 
transferability as more themes and sub-themes could be surfaced were interviews and FGDs able to continue. If given more time and resources, it would have been useful to continue collecting data until data saturation has been reached. However, this study has provided a good scaffolding on stakeholder perspectives that will allow the topic guides to be restructured to cover pertinent points in greater detail.

\section{Conclusions}

This is the first qualitative study examining stakeholders' perspectives on an mHealth intervention in Cambodia. By purposively sampling different levels of stakeholders across the levels of society, the study was able to gather diverse views on the acceptability and feasibility of the Mobile Link project. Clear recommendations have also been provided for interventionists who are considering similar programs, or who are targeting similar hard-toreach populations.

The Mobile Link was perceived to be well-accepted, and well-supported by national and community level stakeholders. This collaborative climate brings hope for implementation of more mHealth interventions in the future. Barriers to successful program implementation (e.g., access of FEWs to health information and services, access of the Mobile Link staff to FEWs, poor rapport with entertainment establishment owners, and low motivation of FEWs) existed at many levels of society and more in-depth investigations will be needed to clarify which should be most urgently addressed. The authors hope that this qualitative analysis of the Mobile Link as a case-study mobile phone based intervention and the recommendations herein will help stakeholders better design programs to maximize the benefits of this type of interventions, and effectively anticipate the challenges to be dealt with.

\section{Acknowledgments}

The authors would like to express their gratitude to the study participants who contributed substantially to the development of the project and field data collection. The authors would also like to thank the staff members of KHANA, and KHANA's implementing partners as well as data collection team. Special thanks go to Ms. Ngovlyly Sok for her support in data collection and transcription.

Funding: None.

\section{Footnote}

Provenance and Peer Review: This article was commissioned by the Guest Editors (Carinne Brody and Sarah Sullivan) for the series "Digital Interventions for Hard-to-reach Populations" published in mHealth. The article was sent for external peer review organized by the Guest Editors and the editorial office.

Conflicts of Interest: All authors have completed the ICMJE uniform disclosure form (available at http:// dx.doi.org/10.21037/mhealth.2020.04.01). The series "Digital Interventions for Hard-to-reach Populations" was commissioned by the editorial office without any funding or sponsorship. The authors have no other conflicts of interest to declare.

Ethical Statement: The authors are accountable for all aspects of the work in ensuring that questions related to the accuracy or integrity of any part of the work are appropriately investigated and resolved. The study was conducted in accordance with the Declaration of Helsinki (as revised in 2013). Ethical approval was obtained from the National Ethics Committee for Health Research (NECHR), Ministry of Health, Cambodia (No. 285NECHR) and the Touro College Institutional Review Board (No. PH-0117), Touro University California, CA, USA. All participants were informed about the study aims and procedures and completed a written informed consent.

Open Access Statement: This is an Open Access article distributed in accordance with the Creative Commons Attribution-NonCommercial-NoDerivs 4.0 International License (CC BY-NC-ND 4.0), which permits the noncommercial replication and distribution of the article with the strict proviso that no changes or edits are made and the original work is properly cited (including links to both the formal publication through the relevant DOI and the license). See: https://creativecommons.org/licenses/by-nc-nd/4.0/.

\section{References}

1. World Health Organization. mHealth: New horizons for health through mobile technologies. Glob Obs eHealth Ser 2011;3:99.

2. Whittaker R, Mcrobbie H, Bullen C, et al. Mobile phonebased interventions for smoking cessation. Cochrane Database Syst Rev 2016;4:CD006611. 
3. Hamine S, Gerth-Guyette E, Faulx D, et al. Impact of mHealth chronic disease management on treatment adherence and patient outcomes: A systematic review. J Med Internet Res 2015;17:e52.

4. Singh P, Panjwani M, Puri CJ, et al. mHealth: Meaning , Purpose and Outcomes. Int J Comput Sci Inf Technol 2016;7:2216-21.

5. Maher L, Dixon TC, Phlong P, et al. Conflicting Rights: How the Prohibition of Human Trafficking and Sexual Exploitation Infringes the Right to Health of Female Sex Workers in Phnom Penh, Cambodia. Health Hum Rights 2015;17:E102-13.

6. Henriquez-Camacho C, Losa J, Miranda JJ, et al. Addressing healthy aging populations in developing countries: Unlocking the opportunity of eHealth and mHealth. Emerg Themes Epidemiol 2014;11:136.

7. Hopkins J. The MAPS Toolkit mHealth Assessment and Planning for Scale Global mHealth Initiative. Geneva: World Health Organization, 2015.

8. Fitzpatrick AL, van Pelt M, Heang H, et al. Using Targeted mHealth Messages to Address Hypertension and Diabetes Self-Management in Cambodia: Protocol for a Clustered Randomized Controlled Trial. JMIR Res Protoc 2019;8:e11614.

9. Choun K, Achanta S, Naik B, et al. Using mobile phones to ensure that referred tuberculosis patients reach their treatment facilities: A call that makes a difference. BMC Health Serv Res 2017;17:575.

10. Smith C, Ngo TD, Gold J, et al. Effect of a mobile phonebased intervention on post-abortion contraception: a randomized controlled trial in Cambodia. Bull World Health Organ 2015;93:842-50A.

11. Sopheab H, Tuot S, Chhea C, et al. Characteristics, risk behaviors and factors associated with abortion among female entertainment workers in Cambodia. Reprod Health 2015;12:82.

12. Yi S, Tuot S, Chhoun P, et al. The impact of a communitybased HIV and sexual reproductive health program on sexual and healthcare-seeking behaviors of female entertainment workers in Cambodia. BMC Infect Dis 2015;15:221.

13. Brody C, Tuot S, Chhoun P, et al. Mobile Link - a theorybased messaging intervention for improving sexual and reproductive health of female entertainment workers in Cambodia: Study protocol of a randomized controlled trial. Trials 2018;19:1-14.

14. Oxfam. Entertainment Workers [Internet]. 2019 [cited 2019 Jun 20]. Available online: https://cambodia.oxfam. org/policy_paper/entertainment-worker

15. Dizechi S, Brody C, Tuot S, et al. Youth paying for sex: What are the associated factors? Findings from a cross-sectional study in Cambodia. BMC Public Health 2018;18:113.

16. Yi S, Chhoun P, Chann N, et al. Prevalence of HIV and Risk Behaviors among Female Entertainment Workers in Cambodia: A National Biological and Behavioral Survey. Am J Public Heal Res 2019;7:94-101.

17. Chhim S, Chhea C, Sopheab H, et al. Proportion and predictors of loss to follow-up in a longitudinal cohort study of female entertainment and sex workers in Cambodia. Int J STD AIDS 2018;29:1295-304.

18. Yi S, Tuot S, Chhoun P, et al. HIV knowledge, HIV testing attitudes and recent HIV testing among female entertainment workers in Cambodia: A cross-sectional study. PLoS One 2018;13:e0198095.

19. Brody C, Chhoun P, Tuot S, et al. HIV risk and psychological distress among female entertainment workers in Cambodia: A cross-sectional study. BMC Public Health 2016;16:133.

20. Coupland H, Page K, Stein E, et al. Structural interventions and social suffering: Responding to amphetamine-type stimulant use among female entertainment and sex workers in Cambodia. Int J Drug Policy 2019;64:70-8.

21. Brody C, Dhaliwal S, Tuot S, et al. Are Text Messages a Feasible and Acceptable Way to Reach Female Entertainment Workers in Cambodia with Health Messages? A Cross-Sectional Phone Survey. JMIR MHealth UHealth 2016;4:e52.

22. Brody C, Tatomir B, Sovannary T, et al. Mobile phone use among female entertainment workers in Cambodia: an observation study. mHealth 2017;3:3.

23. Smith C, Ly S, Uk V, et al. Women's views and experiences of a mobile phone-based intervention to support postabortion contraception in Cambodia. Reprod Health 2017;14:72.

24. Golden SD, Earp JAL. Social Ecological Approaches to Individuals and Their Contexts: Twenty Years of Health Education \& Behavior Health Promotion Interventions. Health Educ Behav 2012;39:364-72.

25. Braun V, Clarke V. Using thematic analysis in psychology. J Chem Inf Model 2013;53:1689-99.

26. Gebru T, Lentiro K, Jemal A. Perceived behavioural predictors of late initiation to HIV/AIDS care in Gurage zone public health facilities: A cohort study using health belief model. BMC Res Notes 2018;11:336. 
27. Svanemyr J, Amin A, Robles OJ, et al. Creating an enabling environment for adolescent sexual and reproductive health: A framework and promising approaches. J Adolesc Health 2015;56:S7-14.

28. van Olmen J, Ku GM, van Pelt M, et al. The effectiveness of text messages support for diabetes self-management: protocol of the TEXT4DSM study in the democratic Republic of Congo, Cambodia and the Philippines. BMC Public Health 2013;13:423.

29. Smith C, Vannak U, Sokhey L, et al. Mobile Technology for Improved Family Planning (MOTIF): The development of a mobile phone-based (mHealth) intervention to support post-abortion family planning (PAFP) in Cambodia. Reprod Health 2016;13:1.

30. Gurupur VP, Wan TTH. Challenges in implementing mHealth interventions: a technical perspective. mHealth 2017;3:32.

31. Siedner MJ, Lankowski A, Musinga D, et al. Optimizing

doi: $10.21037 /$ mhealth.2020.04.01

Cite this article as: Ong KKX, Ng JS, Om C, Chhoun P, Tuot $\mathrm{S}, \mathrm{Yi}$ S. Perceived barriers and facilitators in using text and voice messaging for improving HIV and sexual and reproductive health of female entertainment workers in Cambodia: a qualitative study. mHealth 2020;6:38.
Network Connectivity for Mobile Health Technologies in sub-Saharan Africa. PLoS One 2012; 7:e45643.

32. Zhu A, Tang S, Thu NTH, et al. Analysis of strategies to attract and retain rural health workers in Cambodia, China, and Vietnam and context influencing their outcomes. Hum Resour Health 2019;17:2 .

33. Vu L, Burnett-Zieman B, Banura C, et al. Increasing Uptake of HIV, Sexually Transmitted Infection, and Family Planning Services, and Reducing HIV-Related Risk Behaviors Among Youth Living With HIV in Uganda. J Adolesc Health 2017;60:S22-8.

34. Shannon K, Strathdee SA, Goldenberg SM, et al. Global epidemiology of HIV among female sex workers: Influence of structural determinants. Lancet 2015;385:55-71.

35. Wolf RC, Bingham T, Millett G, et al. Building the evidence base to optimize the impact of key population programming across the HIV cascade. J Int AIDS Soc 2018;21:e25146. 